19. Kennedy PC, Cullen JM, Edwards JF et al (1998) Tumor of the genital system of domestic animals, Bd 4. Armed Forces Institute of Pathology, Washington DC, pp 15-20

20. Denicola DB, Reber AH, Boon GD (1980) Cytology of canine male urogenital tract. Relston Purina, St. Louis

21. Emerson RE, Ulbright TR (2007) Morphological approach to tumors of the testis and paratestis. J Clin Pathol 60:866-880

22. Cheville JC, Sebo TJ, Lager DJ et al (1998) Leydig cell tumor of the testis: a clinicopathological, DNA content and MIB-1 comparison of nonmetastasizing and metastasizing tumors. Ann J Surg Pathol 22:1361-1367

23. Rübben H (2009) Uroonkologie. Springer, Berlin Heidelberg New York

24. Janko AB, Sandberg EC (1970) Histochemical evidence for the protein nature of the Reinke crystalloid. Obstet Gynecol J 30:493-503

25. Remmele W, Klöppel G, Rudolph P et al (2009) Pathologie, Bd 1.Springer, Berlin Heidelberg New York

26. Behrbohm H, Kaschke O, Nawka T (2009) Kurzlehrbuch Hals-Nasen-Ohren-Heilkunde. Thieme, Stuttgart

27. Herxheimer G (1919) Nachruf. Zentralbl Allg Pathol Anat 30:401-403

28. Schumacher GH, Wischhusen H (1970) Anatomia Rostochiensis. Die Geschichte der Anatomie an der 550 Jahre alten Universität Rostock. AkademieVerlag, Berlin

29. Dräger DL, Holstein AF, Wree A (2011) Friedrich Reinke (1862-1919) and his research on human testicles - the Reinke crystals. (28. Arbeitstagung der Anatomischen Gesellschaft 2011). doi:10.3337/anatges.2011.0014

Urologe 2014 · 53:1214 DOI 10.1007/s00120-014-3591-4

Online publiziert: 7. August 2014 C Springer-Verlag Berlin Heidelberg 2014

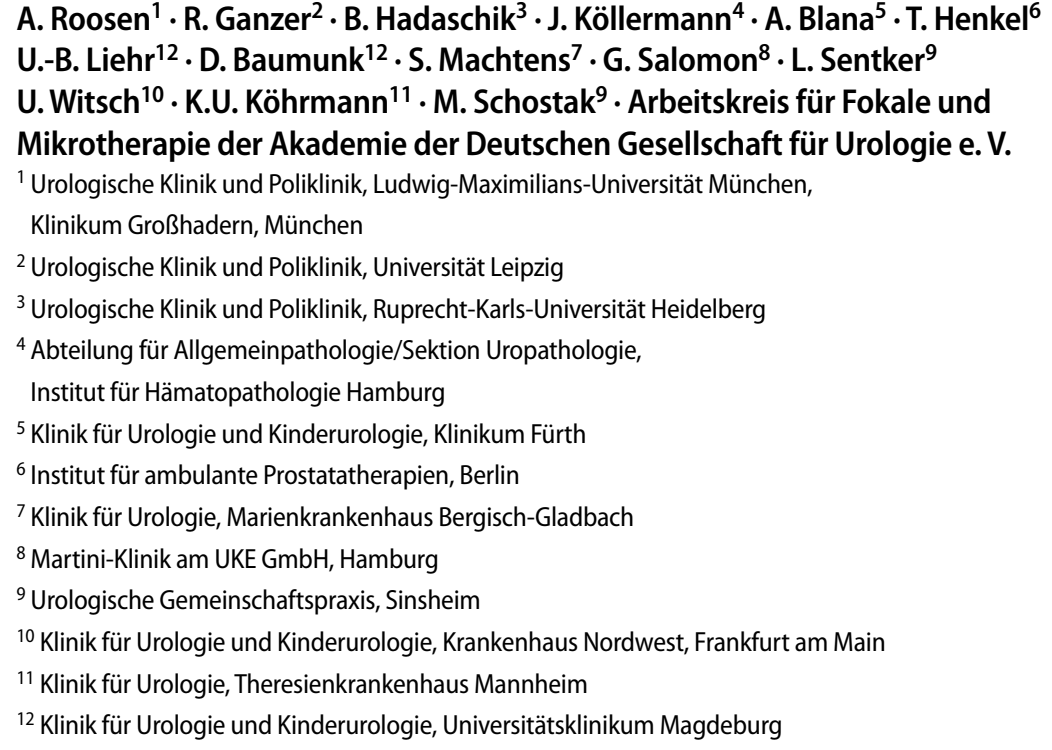

A. Roosen ${ }^{1} \cdot$ R. Ganzer ${ }^{2}$ - B. Hadaschik ${ }^{3} \cdot$ J. Köllermann ${ }^{4} \cdot$ A. Blana ${ }^{5} \cdot$ T. Henkel $^{6}$ U.-B. Liehr ${ }^{12}$. D. Baumunk ${ }^{12}$. S. Machtens ${ }^{7} \cdot$ G. Salomon ${ }^{8} \cdot$ L. Sentker ${ }^{9}$ U. Witsch ${ }^{10} \cdot$ K.U. Köhrmann ${ }^{11} \cdot$ M. Schostak ${ }^{9}$. Arbeitskreis für Fokale und Mikrotherapie der Akademie der Deutschen Gesellschaft für Urologie e.V. ${ }^{1}$ Urologische Klinik und Poliklinik, Ludwig-Maximilians-Universität München, Klinikum Großhadern, München

2 Urologische Klinik und Poliklinik, Universität Leipzig

${ }^{3}$ Urologische Klinik und Poliklinik, Ruprecht-Karls-Universität Heidelberg

${ }^{4}$ Abteilung für Allgemeinpathologie/Sektion Uropathologie, Institut für Hämatopathologie Hamburg

${ }^{5}$ Klinik für Urologie und Kinderurologie, Klinikum Fürth

${ }^{6}$ Institut für ambulante Prostatatherapien, Berlin

${ }^{7}$ Klinik für Urologie, Marienkrankenhaus Bergisch-Gladbach

${ }^{8}$ Martini-Klinik am UKE GmbH, Hamburg

${ }^{9}$ Urologische Gemeinschaftspraxis, Sinsheim

${ }^{10}$ Klinik für Urologie und Kinderurologie, Krankenhaus Nordwest, Frankfurt am Main

${ }^{11}$ Klinik für Urologie, Theresienkrankenhaus Mannheim

${ }^{12}$ Klinik für Urologie und Kinderurologie, Universitätsklinikum Magdeburg

\title{
Erratum zu: Fokale Therapie des Prostatakarzinoms in Deutschland - Status 2014
}

In der HTML-Version des Beitrags ist der Name des Autors U.-B. Liehr nicht korrekt. Die Institutszugehörigkeit von K.U. Köhrmann ist in diesem Beitrag nicht korrekt angegeben. Die Redaktion bittet, die korrekten Angaben zu beachten und die Fehler zu entschuldigen.

\section{Korrespondenzadresse}

\section{A. Roosen}

Urologische Klinik und Poliklinik, Ludwig-Maximilians-Universität München, Klinikum Großhadern,

Marchioninistraße 15, 81377 München alexander.roosen@med.uni-muenchen.de
Die Online-Version des Originalartikels können Sie einsehen unter http://dx.doi.org/10.1007/ s00120-014-3532-2. 